\title{
Nitrogen contents of diamond plates: a comparison of FTIR and SIMS analyses
}

\author{
I. L. Chinn ${ }^{1}$, J. J. Gurney ${ }^{1}$, B Harte ${ }^{2}$, I.C.W. Fitzsimons ${ }^{3}$, and H.J. Milledge ${ }^{4}$
}

1: Dept of Geological Sciences, University of Cape Town, Rondebosch, 7700, South Africa

2: Dept of Geology and Geophysics, Edinburgh University, West Mains Rd, EH9 3JW

3: Dept of Earth Sciences, Monash University, Clayton, Victoria, 3168, Australia

4: Dept of Geological Science, University College London, Gower St, WC1E 6BT, United Kingdom

Nitrogen contents of whole diamonds and polished diamond plates are routinely determined by deconvolution of FTIR (Fourier Transform Infra-red) spectra using experimentally determined absorption coefficients. However, the spatial resolution of this method is limited, (an $80 \mu \mathrm{m}$ aperture is commonly used for macrodiamonds) and measurements in transmission mode result in an average of the absorption characteristics through the thickness of the sample. Integrated FTIR and cathodoluminescence (CL) studies of diamonds have indicated a strong correlation between nitrogen content and luminescence: nitrogen rich zones are characterized by bright blue luminescence, whereas Type II or nitrogen poor zones exhibit dark blue CL.

Complex growth zonation in diamond plates has been recognized from CL photomicrographs, and it was thought that a better understanding of processes occurring during diamond crystallization, such as isotopic fractionation and incorporation of nitrogen defects, would be gained by quantifying nitrogen zonation. In contrast to FTIR spectroscopy, Secondary Ion Mass Spectroscopy (SIMS) is a surface technique with extremely high spatial resolution, and thus provides an excellent means to investigate the relationship between CL features and fine-scale nitrogen variations in polished diamond plates.

The nitrogen contents of diamonds from the George Creek K1 kimberlite dyke in Colorado have been studied previously (Chinn, 1995) using a Bruker IFS45 FTIR spectrometer with microscope attachment and MCT detector cooled by liquid Nitrogen. A sample aperture of $80 \mu \mathrm{m}$ was used for all analyses and spectra were acquired from 200 scans at a resolution of $8 \mathrm{~cm}^{-1}$ over the range 4000 $-650 \mathrm{~cm}^{-1}$. Many of these diamonds consist of complexly intergrown generations of $\mathrm{CO}_{2}$-bearing diamond (with anomalous $\mathrm{CL}$ colours and unusual IR features) and $\mathrm{CO}_{2}$-free diamond (Chinn et al., 1995). The $\mathrm{CO}_{2}$-free diamond exhibits complex growth zonation or evidence of extreme plastic deformation which obscures any primary growth features.

\section{Methods}

Quantitative analysis of spectral data necessitated baseline correction and visual estimation of the percentage of $\mathrm{A}$ and $\mathrm{B}$ nitrogen defects. George Creek diamonds show evidence of A, B and D absorption due to the presence of $\mathrm{A}$ and $\mathrm{B}$ nitrogen aggregates and platelets respectively. No diamonds contained $\mathrm{C}$ absorption features indicative of isolated substitutional nitrogen atoms characteristic of synthetic or metamorphic diamonds. The nitrogen aggregation states of corrected spectra were determined by comparison with reference spectra from synthetic mixtures of pure A and B spectral end members (Mendelssohn and Milledge, 1995).

Nitrogen contents of seven polished diamond plates, mounted in indium and coated with a $\sim 0.02$ $\mu \mathrm{m}$ layer of gold, were measured using the CAMECA ims-4f ion probe at Edinburgh University. The primary ${ }^{133} \mathrm{Cs}^{+}$beam was accelerated at a potential of $+10 \mathrm{kV}$ onto the samples and the secondary ions were accelerated towards the energy filter and mass analyser at a potential of -4.5 $\mathrm{kV}$. Apertures were used to ensure that only the central $8 \mu \mathrm{m}$ of the sputtered area was analysed. Nitrogen abundance was calculated by comparison of ${ }^{12} \mathrm{C}^{14} \mathrm{~N}^{-13} \mathrm{C}^{-}$ratios with a synthetic standard 
(SYNA) of known nitrogen content ( 230 wt ppm $\mathrm{N}$ ) determined by combustion. Nitrogen analyses comprised 50 measurement pairs of the ${ }^{13} \mathrm{C}^{-}$and ${ }^{12} \mathrm{C}^{14} \mathrm{~N}^{-}$peaks for 1 and 2 seconds respectively. In order to monitor any instrument drift with time a few analyses of the synthetic standard were made after not more than 10 analyses of the samples. Regression statistics were used to calculate the ${ }^{12} \mathrm{C}^{14} \mathrm{~N}^{-} /{ }^{13} \mathrm{C}^{-}$ratio of the standard as a function of time and this allowed for calculation of nitrogen abundance in the plates by comparison of ratios. Counting and regression errors were taken into account, and the total error is calculated to be not more than $2 \%$.

\section{Results}

George Creek $\mathrm{CO}_{2}$-free diamonds mostly contain low contents of highly aggregated nitrogen defects or are nitrogen-free (Type II). The maximum nitrogen content measured (expressed in wt ppm) is $2467 \mathrm{ppm}$ and replicate spectra from single stones indicate great heterogeneity in nitrogen content. Spectra indicate the absence of $\mathrm{Ib}$, $\mathrm{IaA}$ or $\mathrm{IaB}$ nitrogen defects in the $\mathrm{CO}_{2}$-bearing diamond generation, however nitrogen may be present in an unknown form not resolvable by the spectrometer.

A strong correlation between $\mathrm{CL}$ and nitrogen content was discovered from the SIMS analyses. $\mathrm{CO}_{2}$-bearing zones with anomalous orange, pink or brown $\mathrm{CL}$ are characterized by low nitrogen contents of below $40 \mathrm{ppm}$. As seen in Fig. 1, plate $\mathrm{GC151}$, which consists solely of $\mathrm{CO}_{2}$-bearing diamond, has a very restricted nitrogen content of $3-5 \mathrm{ppm}$. The $\mathrm{CO}_{2}$-bearing zones of $\mathrm{GC} 150$ and GC171 have higher nitrogen contents of 21-24 and 34-37 ppm respectively. The $\mathrm{CO}_{2}$-free zones of these diamonds span wider individual ranges of 0-16 and 1-22 ppm nitrogen respectively. Another diamond, GC030, has nitrogen contents of 14-22 ppm in $\mathrm{CO}_{2}$-bearing zones, but shows a much wider variation of $0-524 \mathrm{ppm}$ in $\mathrm{CO}_{2}$-free zones, the high nitrogen contents being restricted to narrow growth zones with bright blue $\mathrm{CL}$.

$\mathrm{CO}_{2}$-free diamonds show marked correlation between nitrogen content and intensity of CL. Zones with bright blue CL are characterized by nitrogen contents of $>90-1107 \mathrm{ppm}$, whereas plastically deformed zones and those with dark blue CL have lower nitrogen contents of 0-90 ppm. Sharp discontinuities in nitrogen concentration are associated with CL boundaries: differences of up to $650 \mathrm{ppm}$ over a distance of less than $25 \mu \mathrm{m}$ were found between growth zones.

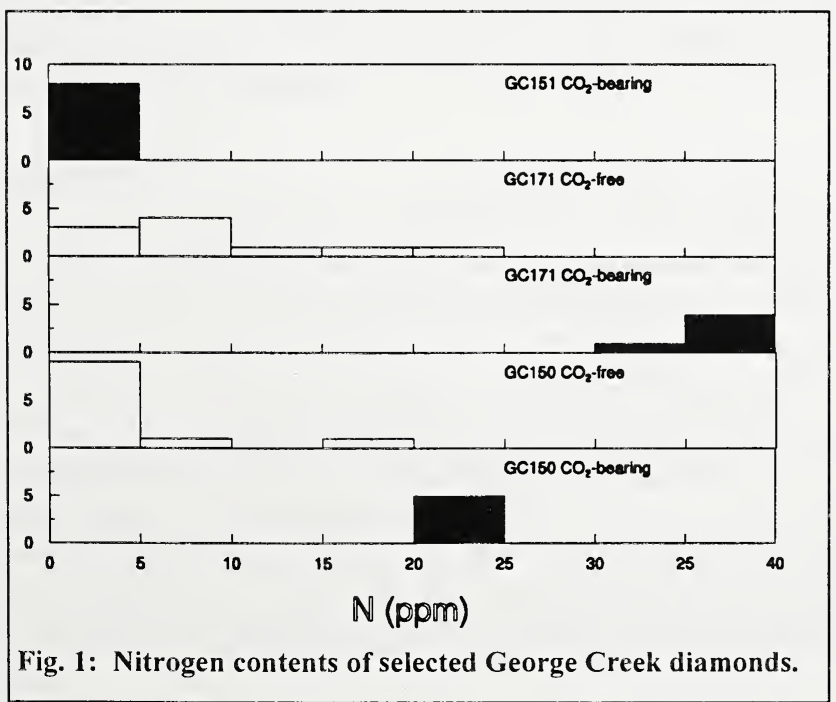

\section{Discussion}

The considerable variation in nitrogen content between growth zones in $\mathrm{CO}_{2}$ free diamonds suggests significant changes in diamond growth conditions with time. These fluctuations may be related to episodic influx of fluids. However, subtle changes in pressure, temperature, oxygen fugacity and other poorly understood factors may have considerable effect on the capacity of the diamond lattice to incorporate nitrogen during crystallization. The low $\mathrm{N}$ content of $\mathrm{CO}_{2}$-bearing growth zones determined by SIMS analyses confirms previous FTIR analyses, and precludes the 
presence of nitrogen as $\mathrm{N}_{2}$ or in defect sites which do not generate IR absorption in the 1-phonon region.

The heterogeneity of nitrogen distribution on the fine scale revealed by SIMS analyses has important implications for the interpretation of FTIR spectroscopy. Most of the nitrogen is hosted in narrow bands which alternate with growth zones depleted in nitrogen. Estimates of the nitrogen content and aggregation state lead to mantle residence time/temperature values which assume a uniform composition over the plate thickness. If zones with highly variable nitrogen content are present, these values will be in error, and in particular a nitrogen-rich zone within nitrogen-poor zones will result in an underestimate of nitrogen content for a given aggregation state, and hence to an overestimate of mantle residence time or temperature.

\section{Acknowledgements}

Polishing of diamond plates was facilitated by M Seal, J.A. Craven is thanked for help with ion probe analyses, and J.W. Harris provided the synthetic diamond standard.

\section{References}

Chinn, I.L. 1995, A study of unusual diamonds from the George Creek K1 kimberlite dyke, Colorado. Unpubl. PhD thesis, University of Cape Town.

Chinn, I.L., Gurney, J.J, Milledge, H.J., Taylor, W.R. and Woods, P.A. 1995, Cathodoluminescence properties of $\mathrm{CO}_{2}$-bearing and $\mathrm{CO}_{2}$-free diamonds from the George Creek K1 kimberlite dike. Intl. Geol. Rev., 37, 254-258.

Mendelssohn, M.J., and Milledge, H.J. 1995, Geologically significant information from routine analysis of the mid Infra-red spectra of diamonds. Intl. Geol. Rev., 37, 95-110. 